\title{
Passivity-Sliding Mode Control of Uncertain Chaotic Systems with Stochastic Disturbances
}

\author{
Zhumu Fu, ${ }^{1,2}$ Leipo Liu, ${ }^{1}$ and Xiaohong Wang ${ }^{1}$ \\ ${ }^{1}$ College of Information Engineering, Henan University of Science and Technology, Luoyang 471023, China \\ ${ }^{2}$ School of Control Science and Engineering, Shandong University, Jinan 250061, China \\ Correspondence should be addressed to Zhumu Fu; fzm1974@163.com
}

Received 22 September 2014; Accepted 9 December 2014; Published 23 December 2014

Academic Editor: Wuneng Zhou

Copyright (C) 2014 Zhumu Fu et al. This is an open access article distributed under the Creative Commons Attribution License, which permits unrestricted use, distribution, and reproduction in any medium, provided the original work is properly cited.

This paper is concerned with the stabilization problem of uncertain chaotic systems with stochastic disturbances. A novel sliding function is designed, and then a sliding mode controller is established such that the trajectory of the system converges to the sliding surface in a finite time. Using a virtual state feedback control technique, sufficient condition for the mean square asymptotic stability and passivity of sliding mode dynamics is derived via linear matrix inequality (LMI). Finally, a simulation example is presented to show the validity and advantage of the proposed method.

\section{Introduction}

Chaos control has received a great deal of interest in the last three decades. Many techniques for chaos control are used, such as adaptive control [1,2], backstepping control [3], fuzzy control [4], and sliding mode control [5-7]. In the above methods, the chaotic system model has a deterministic differential equation; there is no random parameter or random excitation on the system governing equation. But the chaotic system may be affected by stochastic disturbances due to environmental noise $[8,9]$. Stochastic chaotic systems appear in many fields such as chemistry [10], physics and laser science [11], and economics [12]. So it is necessary to study such systems.

There have been some meaningful results about chaos system with stochastic disturbances [13-15]. In [13], the ergodic theory and stochastic noise are considered. Timedelay feedback control of the Van der Pol oscillator under the influence of white noise is investigated in [14]. In [15], an adaptive control of of chaotic systems with white Gaussian noise is considered. On the other hand, sliding mode control is a very effective approach for the robust control systems. It has many attractive features such as fast response, good transient response, and insensitivity to variations. Some related results have been presented [16, 17]. In [16], control of stochastic chaos via sliding mode control is investigated. In [17], chaos synchronization of nonlinear gyros with stochastic excitation is considered by using sliding mode control. Since then, from a practical point of view, many systems need to be passive in order to attenuate noises. So it is necessary to investigate the passivity of chaotic systems via sliding mode control. However, there have been few results in this respect.

Motivated by the above reasons, passivity-sliding mode control problem of uncertain chaotic systems with stochastic disturbances is considered. Stochastic disturbance is a standard Wienner process, the derivative of which generates a continuous time Gaussian white noise. The main contributions of this paper lie in the following aspects. (1) A new sliding function is designed; compared with the existing results $[18,19]$. (2) By incorporating the virtual state feedback control into the performance analysis of the sliding mode dynamics, a sufficient condition for the mean square asymptotic stability and passivity of the sliding mode dynamics is easily derived via LMI. 


\section{Problem Formulation}

Consider the following chaotic system with stochastic disturbances:

$$
\begin{gathered}
d x(t)=[(A+\Delta A) x(t)+B(u(t)+f(x)+\Delta f(x)) \\
+H v(t)] d t+D x(t) d w(t) \\
y(t)=C x(t),
\end{gathered}
$$

where $x(t) \in R^{n}$ is the state vector, $y(t) \in R^{l}$ is the system output, and $u(t) \in R^{m \times n}$ is the control input. $f(x)$ is a nonlinear real-valued function vector. $\Delta f(x)$ represents the uncertainty. $A, B, C, D$, and $H$ are matrices with compatible dimensions, and $(A, B)$ is controllable. $v(t)$ is an external bounded disturbance. $w(t)$ is a one-dimensional Brownian motion. $\Delta A(t)$ is an uncertainty which is assumed to be in the form of

$$
\Delta A(t)=D_{1} F(t) E_{1},
$$

where $D_{1}$ and $E_{1}$ are real matrices with appropriate sizes and $F(t)$ is an unknown time-varying matrix function satisfying

$$
F^{T}(t) F(t) \leq I
$$

The following assumption is necessary for further study.

Assumption 1. The uncertainty $\Delta f(x)$ satisfies

$$
\|\Delta f(x)\| \leq \rho\|x(t)\|,
$$

where $\rho$ is a known positive constant.

\section{Main Results}

For system (1), firstly, a sliding function is designed and a sliding mode controller is designed to drive the state onto the sliding surface in a finite time. Secondly, a sufficient condition is given, which guarantees system (1) on the sliding surface $s(t)=0$ is mean square asymptotically stable and robustly passive.

3.1. Sliding Mode Controller Design. For system (1), a sliding function is designed as

$$
s(t)=B^{T} P\left[x(t)-\eta e^{-\lambda t} x(0)\right]
$$

where $P>0$ is a matrix to be chosen later and $\lambda$ and $\eta$ are positive constants.

Usually, the system (1) on the sliding surface $s(t)=0$ is called the sliding mode dynamics of the system (1).

Remark 2. The sliding function satisfies $s(0)=0$, so the reaching interval is eliminated. Furthermore, if the parameter $\eta=0$, then $s(t)=B^{T} P x(t)$, which is widely used, such as in $[18,19]$.
Definition 3. The sliding mode dynamics of system (1) is said to be robustly passive if there exists a scalar $\gamma>0$ such that

$$
\begin{array}{r}
\mathscr{E}\left\{2 \int_{0}^{t^{*}} v^{T}(s) y(s) d s\right\} \\
\text { for any } v(t) \in \int_{0}^{t^{*}} v^{T}(s) v(s) d s
\end{array}
$$

for all $t^{*} \geq 0$ under zero initial conditions and for all admissible uncertainties.

To achieve the control objective, the input $u(t)$ is designed as follows:

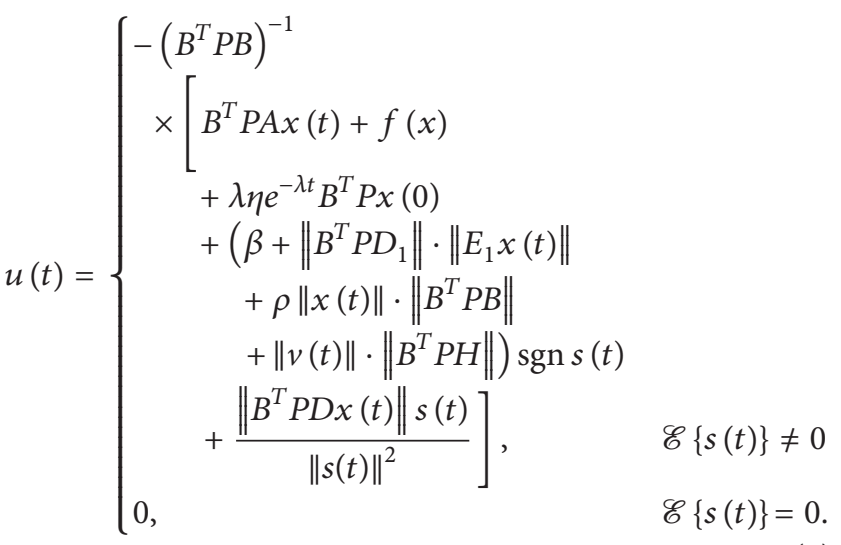

Theorem 4. If the sliding mode controller $u(t)$ is taken as (7), then the trajectory of the system (1) converges to the sliding surface $s(t)=0$ in a finite time.

Proof. Consider a Lyapunov function candidate as follows:

$$
V(t)=s^{T}(t) s(t) .
$$

In fact, we have

$$
\begin{aligned}
\dot{s}(t)= & B^{T} P \dot{x}-\eta e^{-\lambda t} B^{T} P x(0) \\
= & B^{T} P(A+\Delta A) x(t)+B^{T} P B(u(t)+f(x)+\Delta f(x)) \\
& +B^{T} P H v(t)+B^{T} P D x(t) \dot{\omega}(t)+\lambda \eta e^{-\lambda t} B^{T} P x(0) .
\end{aligned}
$$

If $\mathscr{E}\{s(t)\} \neq 0$, calculating the time derivative of $V(t)$ along the trajectory of system (1), then we have

$$
\begin{aligned}
\dot{V}= & 2 s^{T}(t) \dot{s}(t)=2 s^{T}(t) \\
\times & \left(B^{T} P(A+\Delta A) x(t)+B^{T} P B(u(t)+f(x)+\Delta f(x))\right. \\
& \left.+B^{T} P H v(t)+B^{T} P D x(t) \dot{w}(t)+\lambda \eta e^{-\lambda t} B^{T} P x(0)\right) .
\end{aligned}
$$

Substitute (7) into (10), by which the weak infinitesimal operator $\mathscr{L} V(t)$ can be given by

$$
\mathscr{L} V(t) \leq-2 \beta\|s(t)\|
$$


That is,

$$
\mathscr{L} V(t) \leq-2 \beta \sqrt{V(t)}
$$

By Itô's formula, this yields

$$
\mathscr{L}\|s(t)\|=\mathscr{L} \sqrt{V(t)} \leq-\beta
$$

and hence

$$
\mathscr{E}\{\|s(t)\|\} \leq \mathscr{E}\{\|s(0)\|\}-\beta t
$$

which implies $\mathscr{E}\{\|s(t)\|\}$ converges to zero in a finite time. The proof is completed.

3.2. Stability Analysis of the Sliding Mode Dynamics. The following theorem gives a sufficient condition to guarantee the sliding mode dynamics is mean square asymptotically stable and robustly passive.

Theorem 5. Consider system (1) and a given constant $\sigma>0$. If there exist matrices $X>0$ and $Y$ and a scalar $\omega>0$ such that the following linear matrix inequality (LMI) is true:

$$
\left[\begin{array}{cccc}
\bar{\Delta} & H+X C^{T} & X D^{T} & X E_{1}^{T} \\
\star & -\gamma I & 0 & 0 \\
\star & \star & -X & 0 \\
\star & \star & \star & -\oplus I
\end{array}\right]<0,
$$

where $\bar{\Delta}=A X+B Y+X^{T} A^{T}+Y^{T} B^{T}+\omega D_{1} D_{1}^{T}+\sigma X$, then the sliding mode dynamics of system (1) is mean square asymptotically stable and robustly passive. Furthermore, $P=$ $X^{-1}$ and $K=Y X^{-1}$.

Proof. Consider the following Lyapunov functional candidate:

$$
V(t)=x^{T}(t) P x(t) .
$$

The time derivative of $V(t)$ along the trajectories of system (1) is given by

$$
\begin{aligned}
\dot{V}= & 2 x^{T} P[(A+\Delta A) x(t)+B(u(t)+f(x))+H v(t)] \\
& +2 x^{T} P D x(t) \dot{w}(t)+x^{T}(t) D^{T} P D x(t),
\end{aligned}
$$

where $K$ is an arbitrary matrix.

The weak infinitesimal operator $\mathscr{L} V(t)$ can be given by

$$
\begin{aligned}
\mathscr{L} V(t)= & x^{T}(t)\left[P(A+B K)+(A+B K)^{T} P\right] x(t) \\
& +2 x^{T}(t) P \Delta A x(t)+2 x^{T}(t) P H v(t) \\
& +2 x^{T}(t) P B[u(t)+f(x, t)-K x(t)] \\
& +x^{T}(t) D^{T} P D x(t) .
\end{aligned}
$$

Note that

$$
\begin{aligned}
2 x^{T}(t) P \triangle A x(t) \leq & \oplus x^{T}(t) P D_{1} D_{1}^{T} P x(t) \\
& +\frac{1}{\omega} x^{T}(t) E_{1}^{T} E_{1} x(t) .
\end{aligned}
$$

On the sliding surface $s(t)=0$, from (5), we have

$$
2 x^{T}(t) P B=2 \eta e^{-\lambda t} x^{T}(0) P B .
$$

Under the zero-initial conditions, we have

$$
2 x^{T}(t) P B=2 \eta e^{-\lambda t} x^{T}(0) P B=0 .
$$

From (18) and (19), we have

$$
\begin{gathered}
\mathscr{L} V(t)-2 v^{T}(t) y(t)-\gamma v^{T}(t) v(t) \\
\leq\left[\begin{array}{c}
x(t) \\
v(t)
\end{array}\right]^{T}\left[\begin{array}{cc}
\Delta & P H+C^{T} \\
* & -\gamma I
\end{array}\right]\left[\begin{array}{l}
x(t) \\
v(t)
\end{array}\right],
\end{gathered}
$$

where $\Delta=P(A+B K)+(A+B K)^{T} P+\omega P D_{1} D_{1}^{T} P+(1 / \omega) E_{1}^{T} E_{1}+$ $D^{T} P D$.

Before and after multiplying matrix (15) by $\operatorname{diag}\{P, I, P, I\}$, and using $P=X^{-1}$ and $Y=K X$, by Shur complement, we get

$$
\left[\begin{array}{cc}
\Delta+\sigma P & P H+C^{T} \\
* & -\gamma I
\end{array}\right]<0 .
$$

From (22) and (23), we have

$$
\mathscr{L} V(t)-2 v^{T}(t) y(t)-\gamma v^{T}(t) v(t)<-\sigma V(t)<0 .
$$

Integrating (24) from 0 to $t$ and noting that $x(0)=0$, we obtain

$$
0 \leq \mathscr{E}\{V(t)\}<\mathscr{E}\left\{\int_{0}^{t}\left[-\gamma v^{T}(t) v(t)-2 y^{T}(t) y(t)\right] d t\right\} .
$$

We easily get

$$
\mathscr{E}\left\{2 \int_{0}^{t} v^{T}(t) y(t) d t\right\}<\gamma \mathscr{E}\left\{\int_{0}^{t} v^{T}(t) v(t) d t\right\} .
$$

Thus system (1) on the sliding surface $s(t)=0$ is robustly passive.

In fact, when $v(t)=0$, on the sliding surface $s(t)=0$, we can have

$$
\begin{aligned}
\mathscr{L} V(t)= & x^{T}(t)\left[P(A+B K)+(A+B K)^{T} P\right] x(t) \\
& +2 x^{T}(t) P \Delta A x(t)+2 \eta e^{-\lambda t} x^{T}(0) P B \\
& \times[u(t)+f(x, t)-K x(t)]+x^{T}(t) D^{T} P D x(t) .
\end{aligned}
$$

Since $\mathscr{E}\{x(t)\}$ is bounded, then $\mathscr{E}\{u(t)\}$ is bounded from (7). So the term $\mathscr{E}\left\{2 x^{T}(0) P B[u(t)-K x+f(x)]\right\}$ is bounded, where $\mathscr{E}\{\cdot\}$ is an expect operator. Assume that its boundedness is $\delta$.

Noting that (15) implies $\Delta<-\sigma P$, from (27), we have

$$
\mathscr{E}\{\mathscr{L} V(t)\} \leq-\sigma \mathscr{E}\{V(t)\}+\delta e^{-\lambda t} .
$$

Solving (28), we obtain

$$
\begin{aligned}
\mathscr{E}\{V(t)\}<e^{-\sigma t} \mathscr{E}\{V(0)\}+\delta e^{-\sigma t} \int_{0}^{t} e^{(\sigma-\lambda) \tau} d \tau & \\
& = \begin{cases}e^{-\sigma t} \mathscr{E}\{V(0)\}+\delta e^{-\sigma t} t, & \sigma=\lambda, \\
e^{-\sigma t} \mathscr{E}\{V(0)\}+\delta \frac{e^{-\lambda t}-e^{-\sigma t}}{\sigma-\lambda}, & \sigma \neq \lambda .\end{cases}
\end{aligned}
$$


We easily get

$$
\lim _{t \rightarrow \infty} \mathscr{E}\{V(t)\}=0
$$

From the above, we get

$$
\lim _{t \rightarrow \infty} \mathscr{E}\{x(t)\}=0 .
$$

This implies that system (1) with $v(t)=0$ is mean square asymptotically stable on the sliding surface $s(t)=0$. The proof is completed.

Remark 6. From (28), the boundedness of the chaotic system state is used; because the state of chaotic system with stochastic disturbances is indeterminate, the expectation is used to define the boundedness of the state.

\section{Example}

In this section, we use Genesio's chaotic system to show the effectiveness of the method. Genesio's system with stochastic disturbance is as follows:

$$
\begin{aligned}
\dot{x}_{1}(t)= & x_{2}(t)+0.1 x_{1}(t) \dot{w}(t) \\
\dot{x}_{2}(t)= & x_{3}(t)+0.1 x_{3}(t) \dot{w}(t) \\
\dot{x}_{3}(t)= & -6 x_{1}(t)-2.92 x_{2}(t)-1.2 x_{3}(t)+x_{1}^{2}(t) \\
& +0.1 \sin \left(x_{1}(t)\right)+0.001 \sin (t)+u(t), \\
y(t)= & x_{3}(t)
\end{aligned}
$$

where

$$
\begin{gathered}
A=\left[\begin{array}{ccc}
0 & 1 & 0 \\
0 & 0 & 1 \\
-6 & -2.92 & -1.2
\end{array}\right], \quad B=\left[\begin{array}{l}
0 \\
0 \\
1
\end{array}\right], \quad H=\left[\begin{array}{c}
0 \\
0 \\
0.001
\end{array}\right], \\
C=\left[\begin{array}{lll}
0 & 0 & 1
\end{array}\right], \quad D=\left[\begin{array}{ccc}
0.1 & 0 & 0 \\
0 & 0 & 0.1 \\
0 & 0 & 0
\end{array}\right], \quad D_{1}=\left[\begin{array}{ccc}
0.1 & 0 & 0 \\
0 & 0 & 0 \\
0 & 0 & 0
\end{array}\right], \\
E_{1}=0.1, \quad F(t)=\sin t, \quad f(x)=x_{1}^{2}(t), \\
\Delta f(x)=0.1 \cos \left(x_{1}(t)\right), \quad v(t)=\sin (t) .
\end{gathered}
$$

Let $\sigma=0.5$ and $\gamma=0.2$; solving LMI (15) yields

$$
X=\left[\begin{array}{ccc}
1.0290 & -0.3534 & 0.0052 \\
-0.3534 & 0.2286 & -0.1346 \\
0.0052 & -0.1346 & 0.4802
\end{array}\right] \text {. }
$$

Then we have

$$
P=X^{-1}=\left[\begin{array}{ccc}
2.6316 & 4.8524 & 1.3317 \\
4.8524 & 14.1869 & 3.9244 \\
1.3317 & 3.9244 & 3.1684
\end{array}\right] .
$$

The initial value is $x(0)=\left[\begin{array}{lll}-1 & 0 & 1\end{array}\right]^{T}$.

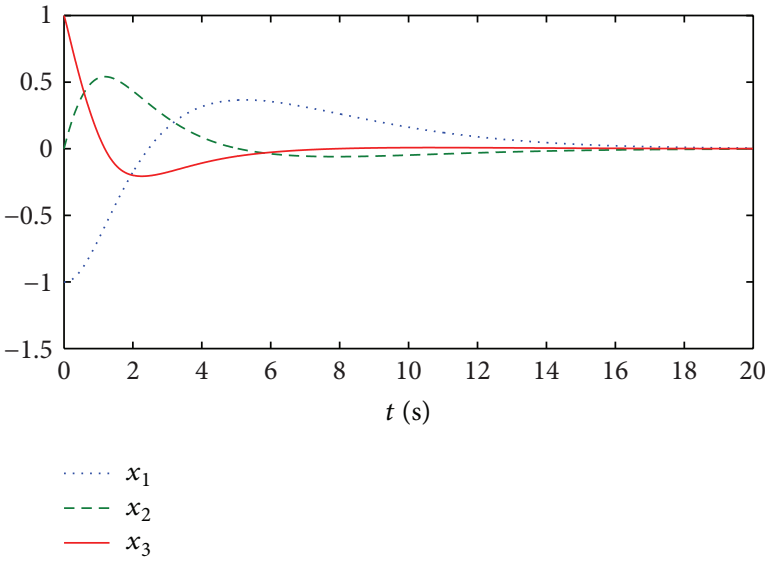

FIgURE 1: State $x(t)$ of the closed-loop system.

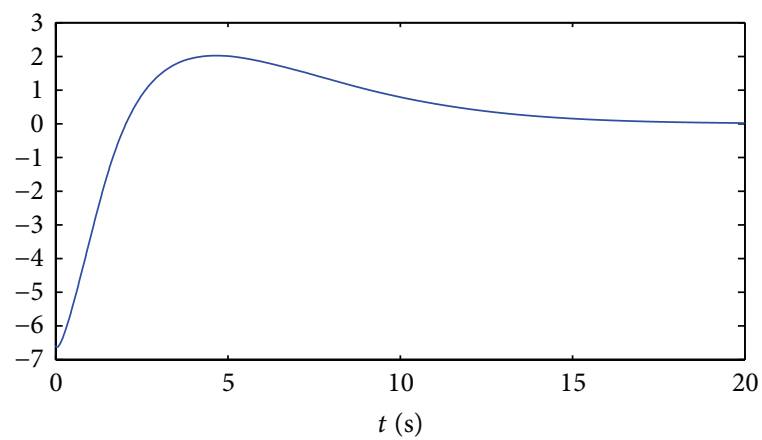

FIGURE 2: The control input $u(t)$.

From Assumption 1, we have $\rho=0.1$. The parameters of controller (7) are $\eta=1$ and $\beta=0.0001$. The simulation results are as shown in Figures 1, 2, 3, and 4.

Figures 1, 2, and 3 show the time responses of the state, control input, and sliding function, respectively. It is concluded that the proposed method is effective. In view of Figure 3 and Figure 4, it is obvious that the sliding function designed in this paper eliminates the reaching interval and reduces the chattering.

\section{Conclusion}

In this paper, the stabilization problem of uncertain chaotic systems with stochastic disturbances is investigated. A new sliding function is proposed, which not only makes the reaching interval eliminated, but also reduces the difficulty of systems analysis and design. A sliding mode controller is designed to make the state of system reach the sliding surface in a finite time. Finally, the simulation shows the effectiveness of the proposed method.

\section{Conflict of Interests}

The authors declare that there is no conflict of interests regarding the publication of this paper. 


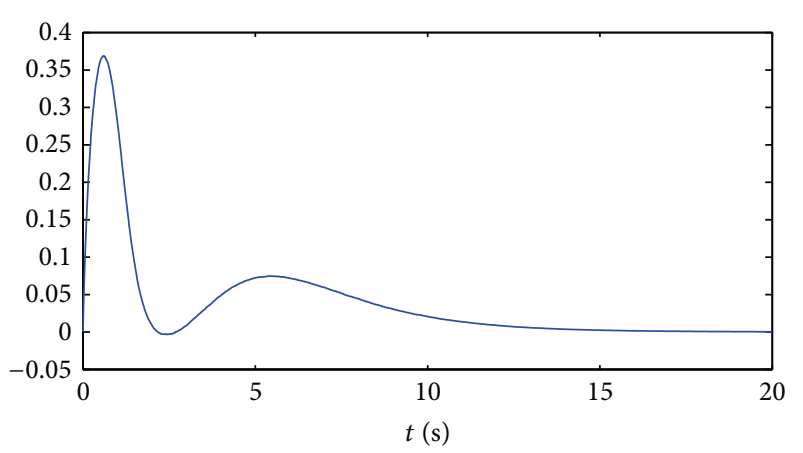

FIGURE 3: The sliding function $s(t)$.

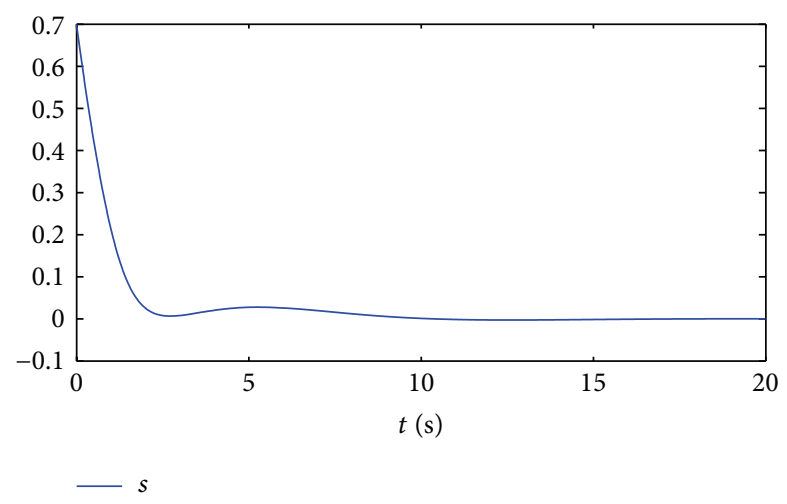

FIGURE 4: The sliding function $s(t)$ in $[18,19]$.

\section{Acknowledgments}

The authors would like to thank the anonymous reviewers for their constructive and insightful comments for further improving the quality of this paper. This work was partially supported by National Natural Science Foundation of China under Grant nos. 61473115, 51277116, and 51375145, China Postdoctoral Science Foundation under Grant no. 2013T60670, Science and Technology Innovative Foundation for Distinguished Young Scholar of Henan Province under Grant no. 144100510004, the Science and Technology Programme Foundation for the Innovative Talents of Henan Province University Grant no. 13HASTIT038, and the Key Scientific and Technological Project of Henan Province Grant no. 132102210247.

\section{References}

[1] C. Hua and X. Guan, "Adaptive control for chaotic systems," Chaos, Solitons \& Fractals, vol. 22, no. 1, pp. 55-60, 2004.

[2] S. Kuntanapreeda and T. Sangpet, "Synchronization of chaotic systems with unknown parameters using adaptive passivitybased control," Journal of the Franklin Institute. Engineering and Applied Mathematics, vol. 349, no. 8, pp. 2547-2569, 2012.

[3] G.-H. Li, "Projective synchronization of chaotic system using backstepping control," Chaos, Solitons and Fractals, vol. 29, no. 2, pp. 490-494, 2006.
[4] J. Wang, G. Qiao, and B. Deng, "Observer-based robust adaptive variable universe fuzzy control for chaotic system," Chaos, Solitons and Fractals, vol. 23, no. 3, pp. 1013-1032, 2005.

[5] H. Wang, X.-L. Zhang, X.-H. Wang, and X.-J. Zhu, "Finite time chaos control for a class of chaotic systems with input nonlinearities via TSM scheme," Nonlinear Dynamics, vol. 69, no. 4, pp. 1941-1947, 2012.

[6] J. Li, W. Li, and Q. Li, "Sliding mode control for uncertain chaotic systems with input nonlinearity," Communications in Nonlinear Science and Numerical Simulation, vol. 17, no. 1, pp. 341-348, 2012.

[7] M. P. Aghababa, S. Khanmohammadi, and G. Alizadeh, "Finitetime synchronization of two different chaotic systems with unknown parameters via sliding mode technique," Applied Mathematical Modelling, vol. 35, no. 6, pp. 3080-3091, 2011.

[8] W. Zhou, D. Tong, Y. Gao, C. Ji, and H. Su, "Mode and delay-dependent adaptive exponential synchronization in pth moment for stochastic delayed neural networks with markovian switching," IEEE Transactions on Neural Networks and Learning Systems, vol. 23, no. 4, pp. 662-668, 2012.

[9] W. Zhou, Q. Zhu, P. Shi, H. Su, J. Fang, and L. Zhou, "Adaptive synchronization for neutraltype neural networks with stochastic perturbation and Markovian switching parameters," IEEE Transactions on Cybernetics, vol. 44, no. 12, pp. 2848-2860, 2014.

[10] M. Pascual and P. Mazzega, "Quasicycles revisited: apparent sensitivity to initial conditions," Theoretical Population Biology, vol. 64, no. 3, pp. 385-395, 2003.

[11] P. R. Patnaik, “The extended Kalman filter as a noise modulator for continuous yeast cultures under monotonic, oscillating and chaotic conditions," Chemical Engineering Journal, vol. 108, no. 1-2, pp. 91-99, 2005.

[12] N. Kikuchi, Y. Liu, and J. Ohtsubo, "Chaos control and noise suppression in external-cavity semiconductor lasers," IEEE Journal of Quantum Electronics, vol. 33, no. 1, pp. 56-65, 1997.

[13] C. Kyrtsou and M. Terraza, "Stochastic chaos or ARCH effects in stock series? A comparative study," International Review of Financial Analysis, vol. 11, no. 4, pp. 407-431, 2002.

[14] E. Scholl, J. Pomplun, A. Amann, A. G. Balanov, and N. B. Janson, "Time-delay feedback control of nonlinear stochastic oscillations," in Proceedings of the 5th EUROMECH Nonlinear Dynamics Conference (ENOC '05), Eindhoven, The Netherlands, August 2005.

[15] H. Salarieh and A. Alasty, "Adaptive control of chaotic systems with stochastic time varying unknown parameters," Chaos, Solitons \& Fractals, vol. 38, no. 1, pp. 168-177, 2008.

[16] H. Salarieh and A. Alasty, "Control of stochastic chaos using sliding mode method," Journal of Computational and Applied Mathematics, vol. 225, no. 1, pp. 135-145, 2009.

[17] H. Salarieh and A. Alasty, "Chaos synchronization of nonlinear gyros in presence of stochastic excitation via sliding mode control," Journal of Sound and Vibration, vol. 313, no. 3-5, pp. 760-771, 2008.

[18] S. Qu and Y. Wang, "Robust control of uncertain time delay system: a novel sliding mode control design via LMI," Journal of Systems Engineering and Electronics, vol. 17, no. 3, pp. 624-628, 2006.

[19] T.-Z. Wu and Y.-T. Juang, "Design of variable structure control for fuzzy nonlinear systems," Expert Systems with Applications, vol. 35, no. 3, pp. 1496-1503, 2008. 

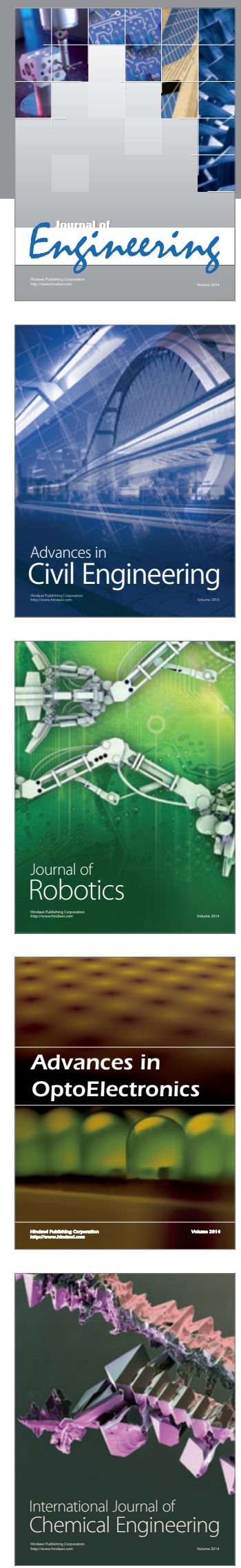

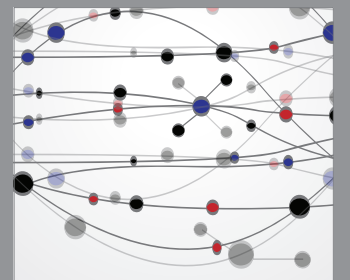

The Scientific World Journal
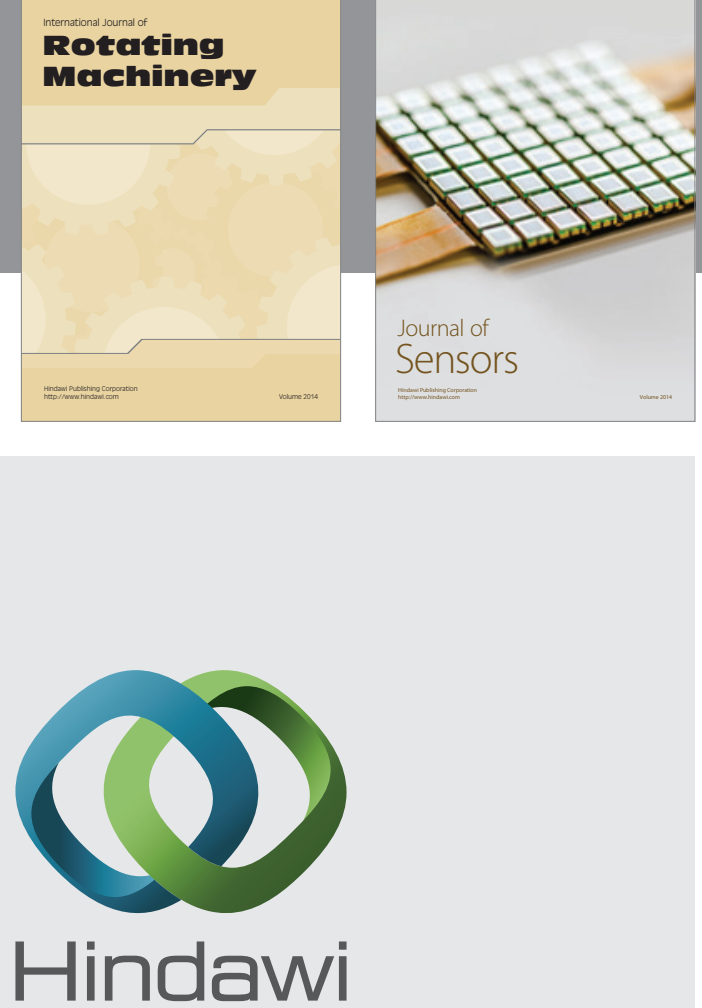

Submit your manuscripts at http://www.hindawi.com
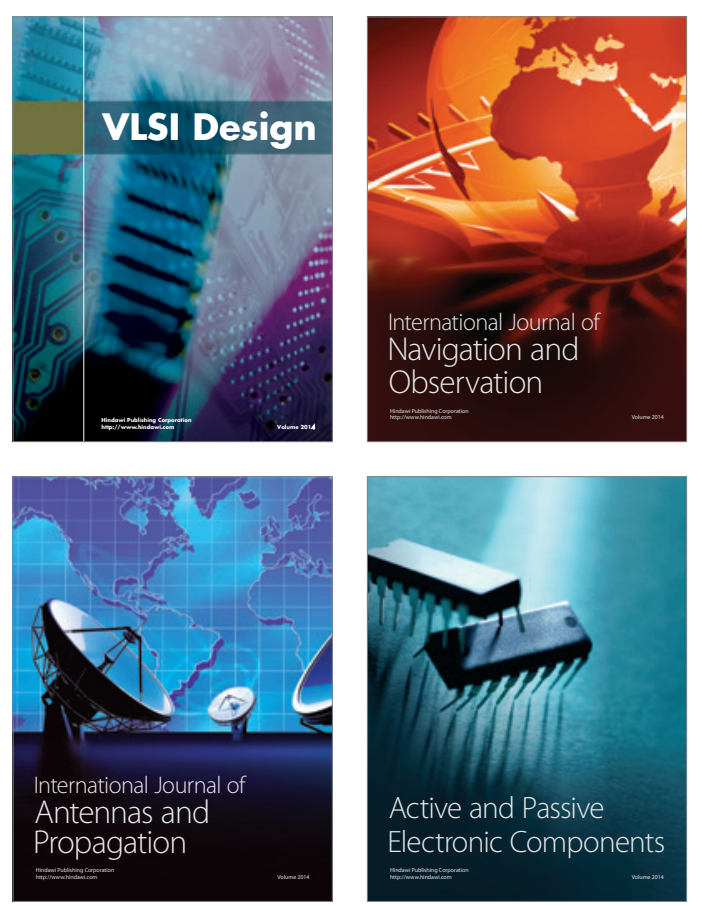
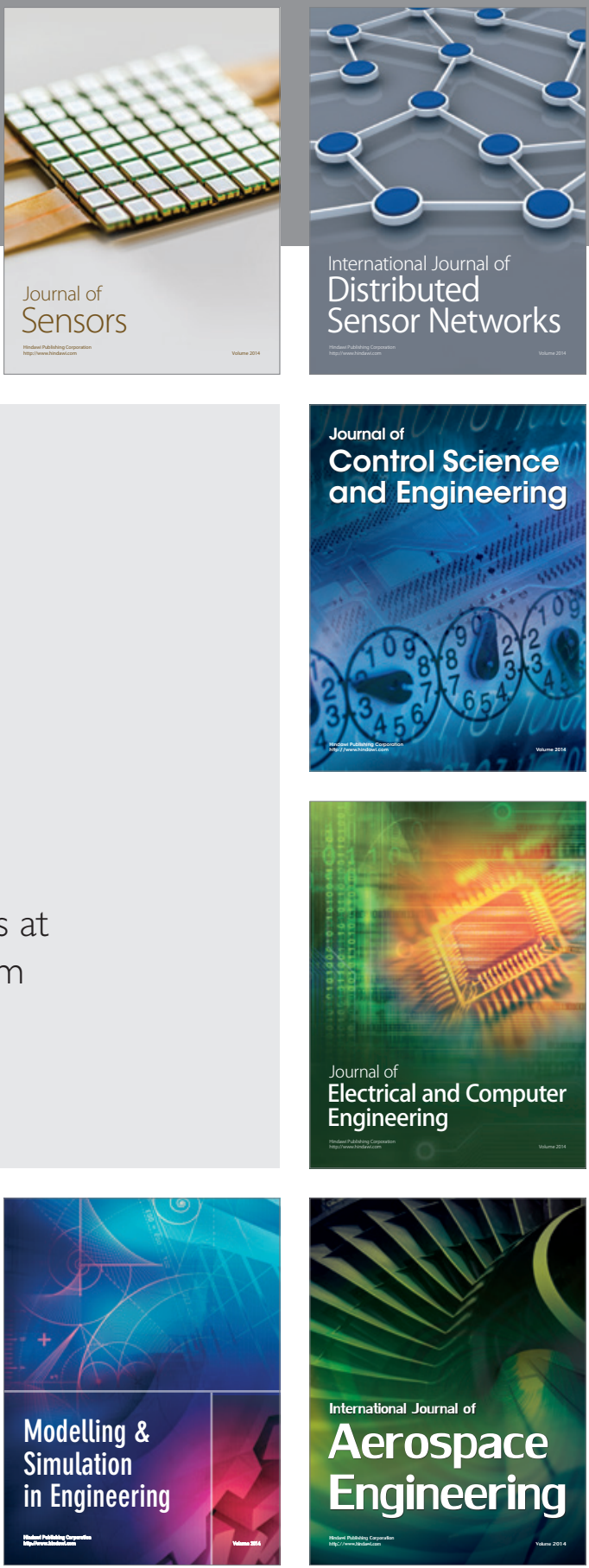

Journal of

Control Science

and Engineering
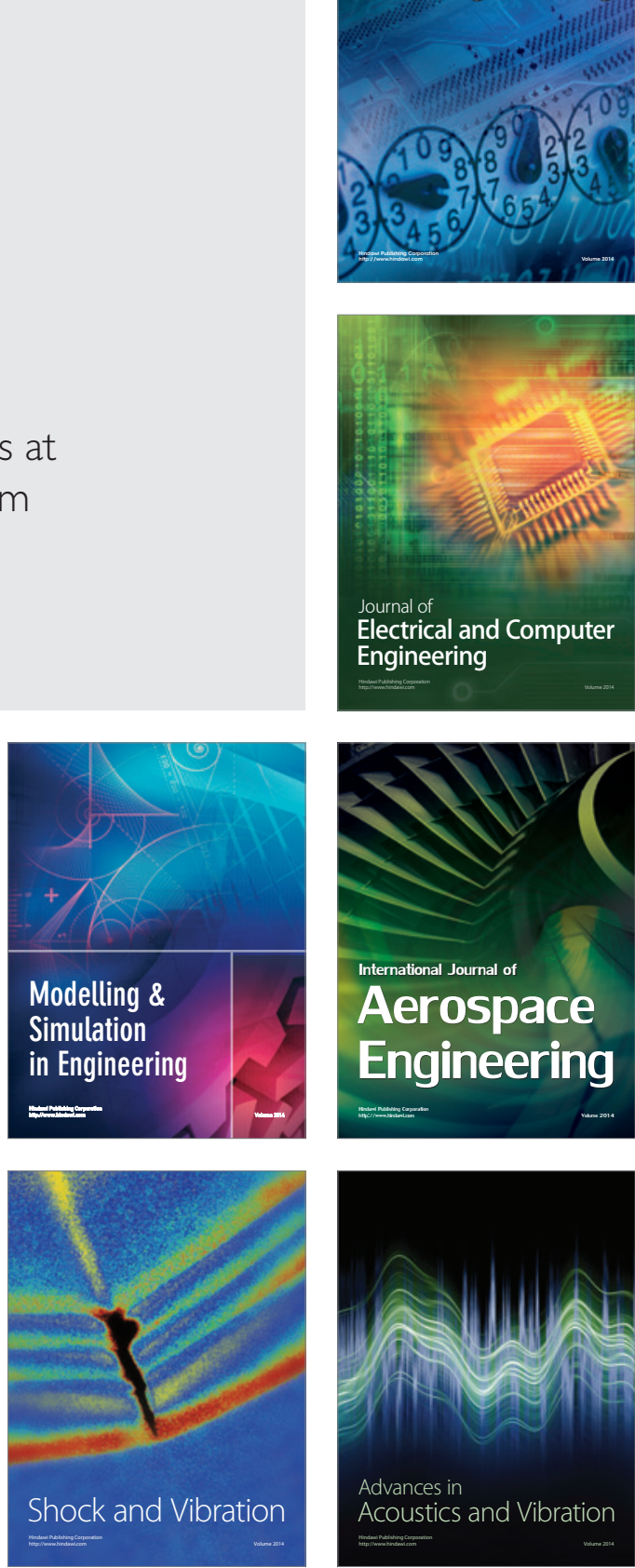